\title{
A generalised Winkler model for the behaviour of shallow foundations
}

\author{
G. T. HOULSBY* ${ }^{*}$ M. J. CASSIDY $\dagger$ and I. EINAV $\dagger$
}

\begin{abstract}
A theoretical model is described for the behaviour of rigid foundations subjected to combined vertical, horizontal and moment loading. The model is based on a generalisation of the Winkler concept of subgrade reaction. Non-linearity of the stress-displacement response is introduced using the concepts of hyperplasticity, and the integration of the stresses over the foundation area is treated within this theoretical framework. The resulting model exhibits (with some limitations) behaviour that quite closely resembles that obtained from experiments and from more sophisticated numerical analyses. The performance of the model is illustrated by calculations for selected load and displacement histories. The primary purpose is to derive models, based on clearly articulated principles, that are capable of describing cyclic behaviour of foundations, and yet retain sufficient simplicity that they can be coupled with analyses of structures. Such models have important applications in the offshore industry for prediction of foundation response under environmental loads.
\end{abstract}

KEYWORDS: bearing capacity; elasticity; footings/foundations; plasticity; theoretical analysis
Nous décrivons un modèle théorique sur le comportement de fondations rigides soumises à une combinaison de charge verticale, horizontale et de moment. Le modèle est basé sur une généralisation du concept de Winkler sur la réaction des fonds de forme. La non linéarité de la réponse contrainte-déplacement est introduite en utilisant les concepts d'hyper-plasticité ; nous traitons l'intégration des contraintes sur la zone de fondation dans ce cadre de travail théorique. Le modèle qui en résulte montre (avec quelques limitations) un comportement qui ressemble d'assez près à celui obtenu par des essais et des analyses numériques plus sophistiquées. Nous illustrons la performance du modèle par des calculs pour des charges et des historiques de déplacement choisis. Le but principal est de dériver des modèles, basés sur des principes clairement exprimés, qui soient capables de décrire le comportement cyclique de fondations tout en restant assez simples pour pouvoir être couplés avec des analyses de structures. Ces modèles ont des applications importantes dans l'industrie offshore où ils servent à prédire la réponse des fondations face à des charges environnementales.

\section{INTRODUCTION}

The theoretical modelling of the behaviour of rigid shallow foundations under combined loading has received considerable attention in recent years. One of the main applications is the modelling of the foundations of offshore structures, in which it is necessary to couple realistic models of the foundation with analyses of the structure.

Two main approaches to foundation modelling can be identified, each with strengths and weaknesses.

(a) Complete analyses of the foundation and soil system, using for instance the finite element method, to model the behaviour of the soil beneath the foundation in detail, and hence model the response of the foundation under any given loading. Such approaches have the advantage that (provided the constitutive model used in the finite element analysis is realistic) they can provide quite realistic modelling of the actual performance of the foundation. The principal disadvantage is that these methods can be computationally extremely time-consuming. Even with modern computing resources they are practicable only for monotonic loading problems, and are not usable for study of cyclic loading. A further disadvantage is that such complete finite element models are rather difficult to couple to a structural analysis package, so that the analyses of foundation and structure are often treated as separate

Manuscript received 26 August 2004; revised manuscript accepted 18 March 2005.

Discussion on this paper closes on 1 February 2006, for further details see p. ii.

* Department of Engineering Science, Oxford University, UK.

$\uparrow$ Centre for Offshore Foundation Systems, the University of Western Australia. exercises. This is inappropriate for problems where there is an interaction between the performance of the structure and of the foundation.

(b) 'Force resultant' models in which the entire behaviour of the foundation is captured in terms of the resultant forces on it, and the corresponding displacements. Such models are typified by Model B and Model C for foundations on clay and sand respectively (Martin \& Houlsby, 2001; Houlsby \& Cassidy, 2002). These models are usually framed in terms of hardening plasticity theory, and employ a yield surface to describe the limiting load combinations that can be applied to the footing. They have the advantage that (by comparison with (a) above) they are much more compact numerically, and require only a tiny fraction of the computational effort. With careful calibration against physical and numerical experiments they can provide a good description of the foundation behaviour, and can readily be incorporated in structural analyses. Their main disadvantage is that, in avoiding modelling of the soil beneath the structure, they inevitably lead to some loss of detail.

Models using approach (b) above were first developed to describe monotonic loading of foundations, and usually employ a single yield surface. These models do not provide very realistic modelling of cyclic behaviour. To extend their applicability to cyclic loading, multiple yield surfaces (or even an infinite number of yield surfaces, which can be accommodated within the continuous hyperplasticity framework; Puzrin \& Houlsby, 2001) of different sizes are required. One promising approach is to scale the single yield surface to create many nested surfaces (illustrated schematically as the transition from Fig. 1(a) to Fig. 1(b)), and this may allow realistic modelling of cyclic behaviour for combi- 
H

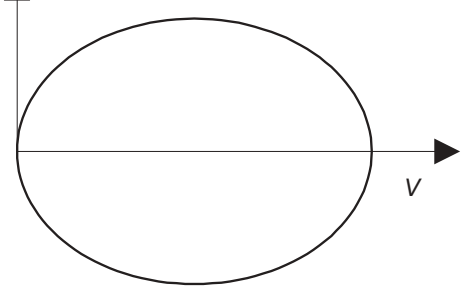

(a)

$H$

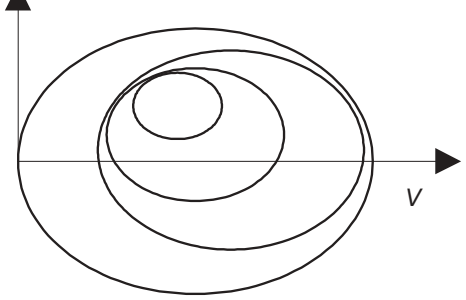

(b)

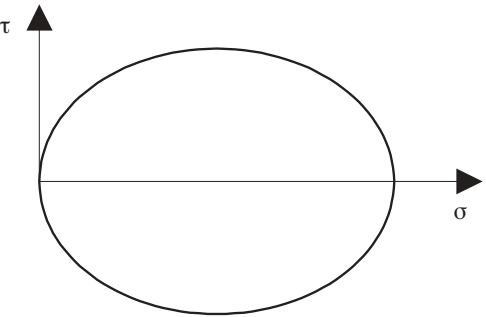

(c)

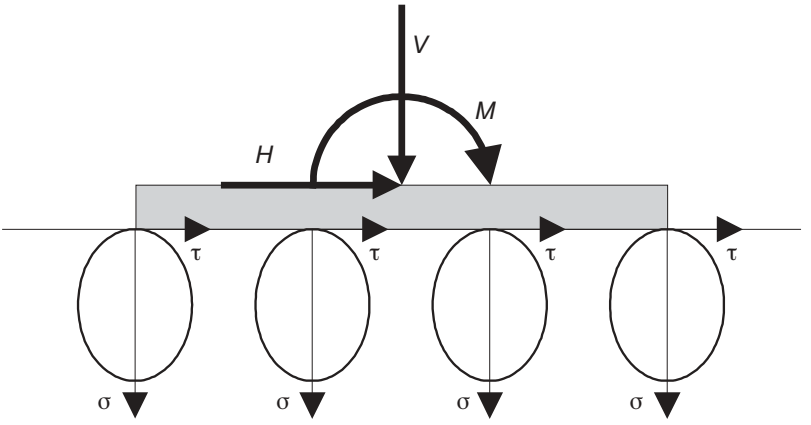

(d)

Fig. 1. Generalisation of single-surface to multi-surface plasticity models: (a) single-surface model in load space; (b) multiple-surface model in load space; (c) single-surface model in stress space; (d) integration of stress-space models over area of foundation $(V=$ vertical load, $H=$ horizontal load, $M=$ overturning moment, $\sigma=$ normal traction on foundation, $\tau=$ shear traction on foundation)

nation with structural analyses. However, the calibration of such models against experimental data is a rather more complex exercise than for the single yield surface models. A disadvantage of this approach to development of multiple surface models is that it is difficult to ascribe a simple physical meaning to the yield surfaces; they are essentially just mathematical abstractions, and it is only the overall behaviour predicted by the model that can be interpreted physically.

In this paper we explore an alternative approach that in effect falls between the levels of detail employed in $(a)$ and (b) above. It involves multiple yield surfaces (or an infinite number of yield surfaces), each representing the behaviour of a point on the footing. Conceptually this is illustrated by first expressing a single yield surface model in terms of the normal and shear stresses $\sigma, \tau$ at points on the foundation rather than total forces (Fig. 1(a)-(c)), and then applying this model for each point across the base of the footing (Fig. 1(d)). In contrast with the use of yield surfaces in force resultant space, as in Fig. 1(b), the physical interpretation is much more straightforward. An advantage is that the calibration process is rather simpler, as the physical meanings that can be ascribed to parameters allow sensible values to be selected more easily. The resulting models are sufficiently straightforward that they can readily be combined with structural analyses of cyclic loading problems. Thus they manage to circumvent some of the disadvantages of both approach $(a)$ and approach $(b)$ above.

The models are based on a generalisation of the Winkler concept of subgrade reaction, a methodology also explored for combined loading problems by Einav \& Cassidy (2004) and Einav (2004). In Winkler methods it is generally assumed that, for each point on a foundation, there is a linear (elastic) relationship between the traction (force per unit area) developed on the foundation and the displacement at that point. Importantly, there is no interaction assumed between the displacement at one point and the traction at another. It is well known that such an approximation has severe limitations, because in practice such interactions play an important role in the response of the foundation. In the following, however, we introduce non-linearities in the pointwise response that, to a certain extent, avoid some of these limitations.

In this paper we generalise the Winkler concept so that at each point an elastic-plastic response is defined, but again there is no interaction between points. Such a generalisation is of course routinely used in the $p-y$ and $t-z$ analysis methods for lateral and axial loading of piles (e.g. Fleming et al., 1985), but is much less common in the analysis of shallow foundations. There are two principal components to the model: we define $(a)$ the behaviour at each point on the foundation, and $(b)$ the integration of the behaviour over the base of the foundation to produce the entire model. We approach the pointwise model by using the hyperplasticity approach, based on the formulation defined by Houlsby \& Puzrin (2000). The integration over the base of the foundation is then a simple application of the continuous hyperplasticity approach described by Puzrin \& Houlsby (2001). Both of these methods place strong emphasis on the use of two potentials: one defining the stored energy, and one defining the rate of dissipation. A full description of hyperplasticity is not possible here, but a brief summary of the key equations and references is given in Appendix 1.

\section{MODEL DEVELOPMENT \\ The pointwise model}

Vertical loading. We consider first purely vertical loading, and define the relationship between the normal traction $\sigma$ (positive in compression) and the vertical displacement $v$ (positive downwards) at any point on a footing of width $B$ on a soil with a shear modulus $G$ (see Fig. 2). The elastic relationship is $\sigma=\left(k_{\mathrm{v}} G / B\right) v$, where $k_{\mathrm{v}} G / B$ is the elastic 


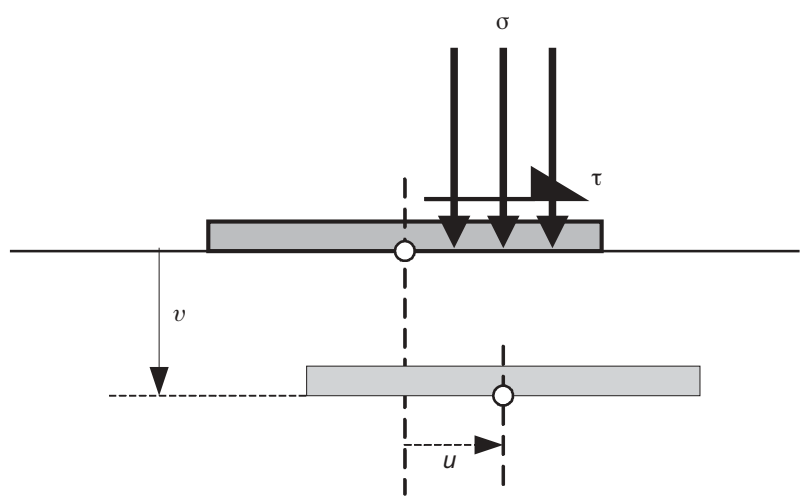

Fig. 2. Normal and shear traction on a footing

coefficient of subgrade reaction and $k_{\mathrm{v}}$ is a dimensionless factor.

In the hyperplastic approach this behaviour is defined by specifying a free energy (per unit area) $f=\left(k_{\mathrm{v}} G / 2 B\right) v^{2}$, and then using the relationship $\sigma=(\partial f / \partial v)=\left(k_{\mathrm{v}} G / B\right) v$. The behaviour is shown schematically in Fig. 3(a).

The first modification we consider is that of contactbreaking. If the foundation is subjected to compression then the elastic relationship defined above applies, but if an attempt is made to apply tension then the foundation simply lifts off from the soil with no applied load. This is achieved by modifying the expression for $f$ to $f=\left(k_{\mathrm{v}} G / 2 B\right)\langle v\rangle^{2}$, where \langle\rangle are Macaulay brackets such that $\langle x\rangle=0, x<0 ;\langle x\rangle=x$, $x \geqslant 0$. This results in $\sigma=\left(k_{\mathrm{v}} G / 2 B\right)\langle v\rangle$, so that for $v$ positive the relationship is as before, and for $v$ negative the stress is zero, as it would be when contact is broken. The response is as in Fig. 3(b).

Alternatively one may introduce plasticity by defining a vertical plastic displacement $\alpha$. The energy expression now becomes $f=\left(k_{\mathrm{v}} G / 2 B\right)(v-\alpha)^{2}$, and this is supplemented by an expression for the dissipation rate (per unit area) $d=N_{\mathrm{v}} c|\dot{\alpha}|$, where $c$ is the shear strength of the soil and $N_{\mathrm{v}}$ is a dimensionless factor. We use $c$ here because we shall address just total stress models in which the undrained strength is appropriate. It is straightforward to show, by applying the formulation of Houlsby \& Puzrin (2000), that this leads to yield at $\sigma= \pm N_{\mathrm{v}} c$ (so that the foundation has equal capacities in tension and compression), and the behaviour is as in Fig. 3(c). Note that in the approach we adopt, once the dissipation is specified, the yield condition follows as a result. This is in contrast to the more conventional approach in which the yield condition is specified directly. Once familiarity is gained with the hyperplastic approach it is possible to choose appropriate dissipation functions to define particular yield conditions.

The two phenomena of contact-breaking and yield can be combined in a single model by introducing the modification $f=\left(k_{\mathrm{v}} G / 2 B\right)\langle v-\alpha\rangle^{2}$. The response is then as in Fig. 3(d). Note that after a large loading-unloading path $A B C D E$ the reloading would be back along EDC, as the contact would not be re-established until point $\mathrm{D}$.

Table 1 shows the development of the models for vertical loading in the terminology used by Houlsby \& Puzrin (2000), and clearly shows the relationships between the different possible models of behaviour. Note that only one of the potentials $d$ or $y$ (or, for the rate dependent case $z$ or $w$ ) needs to be defined as, in principle, the other can then be

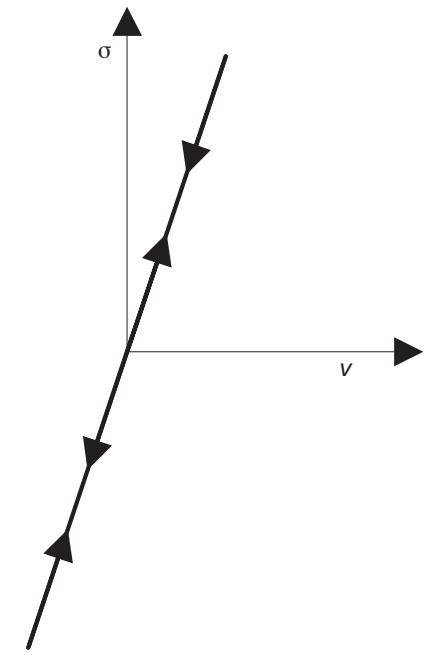

(a)

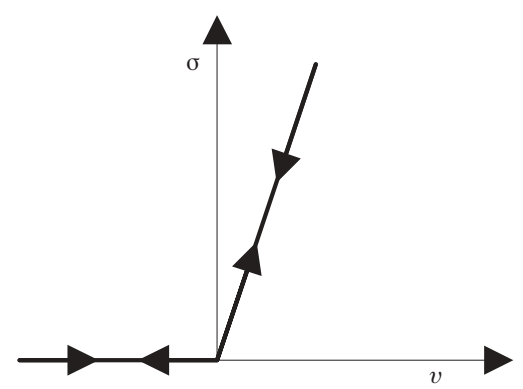

(b)

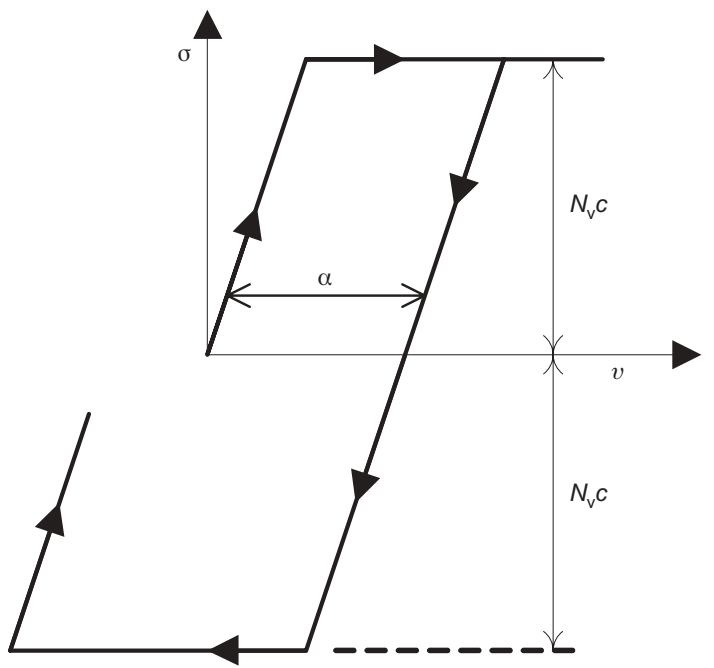

(c)

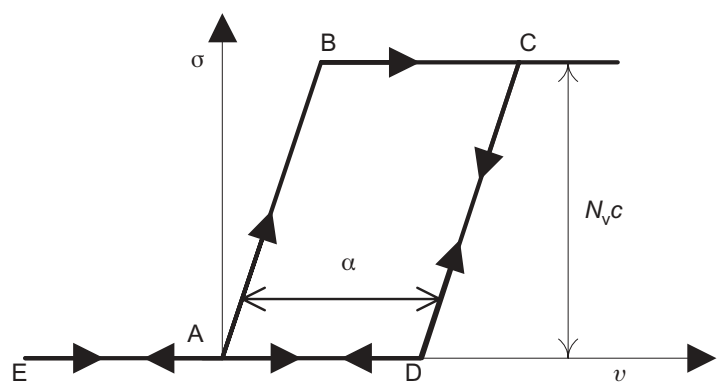

(d)

Fig. 3. Alternative forms of model under vertical loading: (a) elastic; (b) elastic, contact-breaking; (c) elastic-plastic; (d) elastic-plastic, contact-breaking 
Table 1. Alternative pointwise models for vertical loading

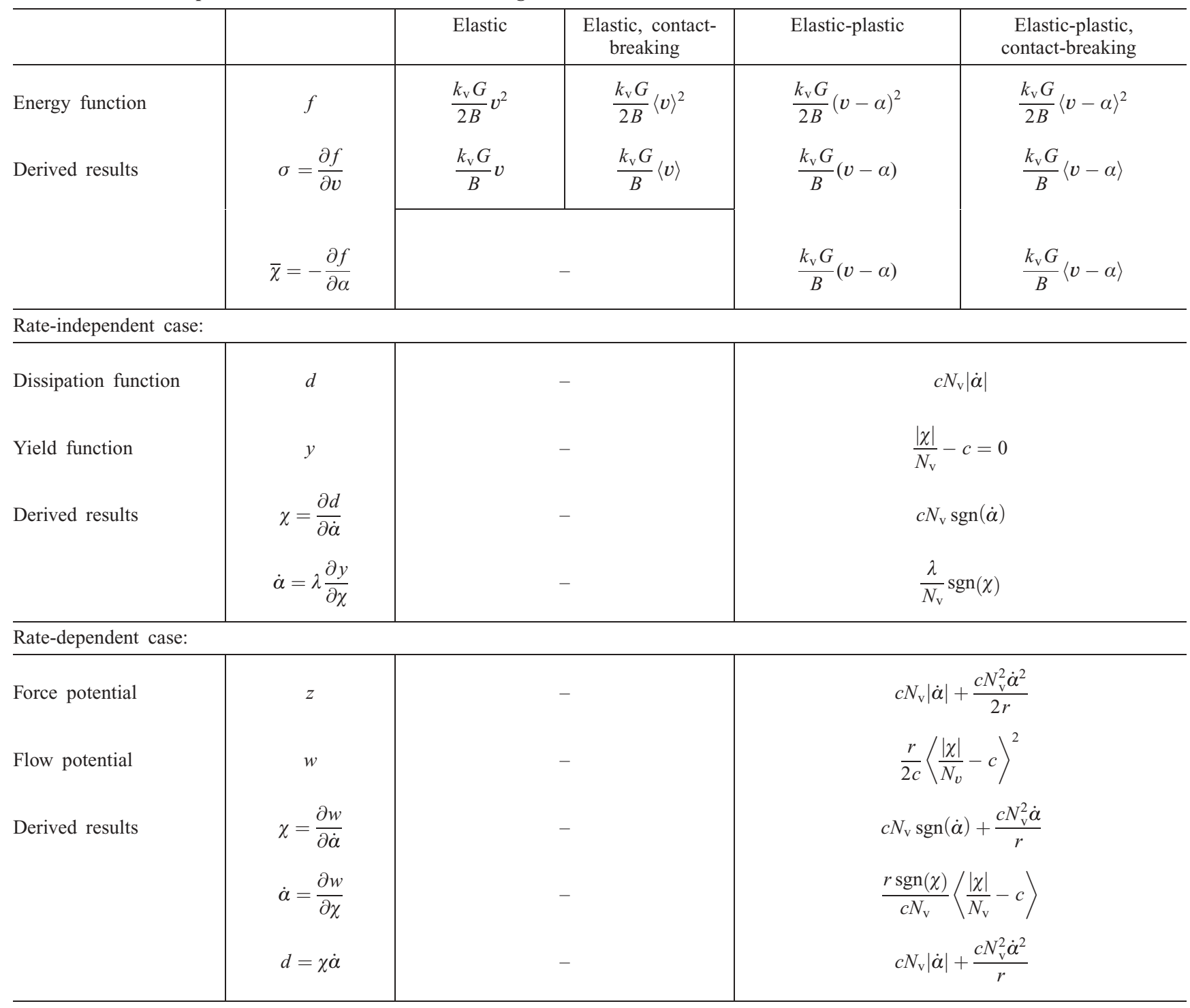

determined. Also shown are the derived results, both for the rate-independent case (as discussed above) and also a ratedependent variation, based on the formulation of Houlsby \& Puzrin (2002), which is more useful for numerical analysis. The latter involves a rate dependence parameter $r$ with dimensions of $\dot{v}$. The rate independent solution is recovered as $r \rightarrow \infty$.

Vertical and horizontal loading. We generalise the elastic relationship to vertical and horizontal loading (see Fig. 2) by writing $f=\left(k_{\mathrm{v}} G / 2 B\right) v^{2}+\left(k_{\mathrm{h}} G / 2 B\right) u^{2}$, leading to $\sigma=$ $\left(k_{\mathrm{v}} G / B\right) v$ and $\tau=\left(k_{\mathrm{h}} G / B\right) u$.

The generalisation of the contact-breaking phenomenon is more complex, as it is important that the model results in the shear stress as well as the vertical stress falling to zero as contact is lost. The most satisfactory way to implement this is to treat contact-breaking as a special case of frictional behaviour. This is achieved by introducing a variable $\beta_{\mathrm{h}}$, which is the magnitude of the horizontal sliding. The free energy becomes $f=\left(k_{\mathrm{v}} G / 2 B\right)\langle v\rangle^{2}+\left(k_{\mathrm{h}} G / 2 B\right)\left(u-\beta_{\mathrm{h}}\right)^{2}$, and there is a frictional dissipation $d=\mu \sigma\left|\dot{\beta}_{\mathrm{h}}\right|$, where $\mu$ is a frictional constant. If $v$ is positive then sliding occurs at $|\tau|=\mu \sigma$. If $v$ is negative (and contact is lost) then $\sigma$ is zero. It follows that $\dot{\beta}_{\mathrm{h}}=\dot{u}$ and that the footing can be displaced sideways under no force. Thus the model correctly describes the possibility that the footing can be picked up and then replaced (stress-free) at a different location.

The plasticity can simply be generalised by including both horizontal and vertical plastic deformation, with the dissipation being a combination of the two terms: for example $f$ becomes $f=\left(k_{\mathrm{v}} G / 2 B\right)\left(v-\alpha_{\mathrm{v}}\right)^{2}+\left(k_{\mathrm{h}} G / 2 B\right)\left(u-\alpha_{\mathrm{h}}\right)^{2}$ and $d$ becomes $d=c \sqrt{N_{\mathrm{v}}^{2} \dot{\alpha}_{\mathrm{v}}^{2}+N_{\mathrm{h}}^{2} \dot{\alpha}_{\mathrm{h}}^{2}}$. (Note that we use $\alpha$ variables for plastic deformation and $\beta$ variables for sliding: in particular there are now two irrecoverable components of horizontal movement, $\alpha_{\mathrm{h}}$ and $\beta_{\mathrm{h}}$.) For this case it is straightforward to show that yield occurs on the elliptical surface:

$$
\left(\frac{\sigma}{N_{\mathrm{v}}}\right)^{2}+\left(\frac{\tau}{N_{\mathrm{h}}}\right)^{2}=c^{2}
$$

The model that we pursue in the following combines contact-breaking with yield, and is fully defined by the following equations:

$$
\begin{aligned}
& f=\frac{k_{\mathrm{v}} G}{2 B}\left\langle v-\alpha_{\mathrm{v}}\right\rangle^{2}+\frac{k_{\mathrm{h}} G}{2 B}\left(u-\alpha_{\mathrm{h}}-\beta_{\mathrm{h}}\right)^{2} \\
& d=c \sqrt{N_{\mathrm{v}}^{2} \dot{\alpha}_{\mathrm{v}}^{2}+N_{\mathrm{h}}^{2} \dot{\alpha}_{\mathrm{h}}^{2}}+\mu \sigma\left|\dot{\beta}_{\mathrm{h}}\right|
\end{aligned}
$$

from which the following can be derived: 


$$
\begin{aligned}
& \sigma=\frac{\partial f}{\partial v}=\frac{k_{\mathrm{v}} G}{B}\left\langle v-\alpha_{\mathrm{v}}\right\rangle \\
& \tau=\frac{\partial f}{\partial u}=\frac{k_{\mathrm{h}} G}{B}\left(u-\alpha_{\mathrm{h}}-\beta_{\mathrm{h}}\right) \\
& \bar{\chi}_{\alpha_{\mathrm{v}}}=-\frac{\partial f}{\partial \alpha_{\mathrm{v}}}=\frac{k_{\mathrm{v}} G}{B}\left\langle v-\alpha_{\mathrm{v}}\right\rangle=\sigma \\
& \bar{\chi}_{\alpha_{\mathrm{h}}}=-\frac{\partial f}{\partial \alpha_{\mathrm{h}}}=\frac{k_{\mathrm{h}} G}{B}\left(u-\alpha_{\mathrm{h}}-\beta_{\mathrm{h}}\right)=\tau \\
& \bar{\chi}_{\beta_{\mathrm{h}}}=-\frac{\partial f}{\partial \beta_{\mathrm{h}}}=\frac{k_{\mathrm{h}} G}{B}\left(u-\alpha_{\mathrm{h}}-\beta_{\mathrm{h}}\right)=\tau \\
& \chi_{\alpha_{\mathrm{v}}}=\frac{\partial d}{\partial \dot{\alpha}_{\mathrm{v}}}=\frac{c N_{\mathrm{v}}^{2} \dot{\alpha}_{\mathrm{v}}}{\sqrt{N_{\mathrm{v}}^{2} \dot{\alpha}_{\mathrm{v}}^{2}+N_{\mathrm{h}}^{2} \dot{\alpha}_{\mathrm{h}}^{2}}} \\
& \chi_{\alpha_{\mathrm{h}}}=\frac{\partial d}{\partial \dot{\alpha}_{\mathrm{h}}}=\frac{c N_{\mathrm{h}}^{2} \dot{\alpha}_{\mathrm{h}}}{\sqrt{N_{\mathrm{v}}^{2} \dot{\alpha}_{\mathrm{v}}^{2}+N_{\mathrm{h}}^{2} \dot{\alpha}_{\mathrm{h}}^{2}}} \\
& \chi_{\beta_{\mathrm{h}}}=\frac{\partial d}{\partial \dot{\beta}_{\mathrm{h}}}=\mu \sigma \operatorname{sgn}\left(\dot{\beta}_{\mathrm{h}}\right)
\end{aligned}
$$

where $\operatorname{sgn} x=-1$ if $x<0, \operatorname{sgn} x=1$ if $x>0$, and $\operatorname{sgn} x$ is undefined (but in the range $-1 \leqslant \operatorname{sgn} x \leqslant 1$ ) at $x=0$. When combined with the conditions $\bar{\chi}=\chi$ for each of the relevant subscripted variables (see Houlsby \& Puzrin, 2000) the above equations provide a complete description of the mechanical behaviour of the system. The following yield criteria can be derived:

$$
\begin{aligned}
& y_{\alpha}=\sqrt{\left(\frac{\chi_{\alpha_{\mathrm{v}}}}{N_{\mathrm{v}}}\right)^{2}+\left(\frac{\chi_{\alpha_{\mathrm{h}}}}{N_{\mathrm{h}}}\right)^{2}}-c=0 \\
& y_{\beta}=\left|\chi_{\beta_{\mathrm{h}}}\right|-\mu \sigma=0
\end{aligned}
$$

Noting the relationships between the generalised stresses $\chi=\bar{\chi}$ and the true stresses, these are equivalent to equation (1) and the frictional relationship $|\tau|=\mu \sigma$. The yield surfaces are shown in Fig. 4, in which the accessible region of stress space is shown shaded. From the above one can derive

$$
\begin{aligned}
& \dot{\alpha}_{\mathrm{v}}=\lambda_{\alpha} \frac{\partial y_{\alpha}}{\partial \chi_{\alpha_{\mathrm{v}}}}=\lambda_{\alpha} \frac{\chi_{\alpha_{\mathrm{v}}} / N_{\mathrm{v}}^{2}}{\sqrt{\left(\chi_{\alpha_{\mathrm{v}}} / N_{\mathrm{v}}\right)^{2}+\left(\chi_{\alpha_{\mathrm{h}}} / N_{\mathrm{h}}\right)^{2}}} \\
& \dot{\alpha}_{\mathrm{h}}=\lambda_{\alpha} \frac{\partial y_{\alpha}}{\partial \chi_{\alpha_{\mathrm{h}}}}=\lambda_{\alpha} \frac{\chi_{\alpha_{\mathrm{h}}} / N_{\mathrm{h}}^{2}}{\sqrt{\left(\chi_{\alpha_{\mathrm{v}}} / N_{\mathrm{v}}\right)^{2}+\left(\chi_{\alpha_{\mathrm{h}}} / N_{\mathrm{h}}\right)^{2}}} \\
& \dot{\beta}_{\mathrm{h}}=\lambda_{\beta} \frac{\partial y_{\beta}}{\partial \chi_{\beta_{\mathrm{h}}}}=\lambda_{\beta} \operatorname{sgn}\left(\chi_{\beta_{\mathrm{h}}}\right)
\end{aligned}
$$

Finally we note that for computational purposes it is con-

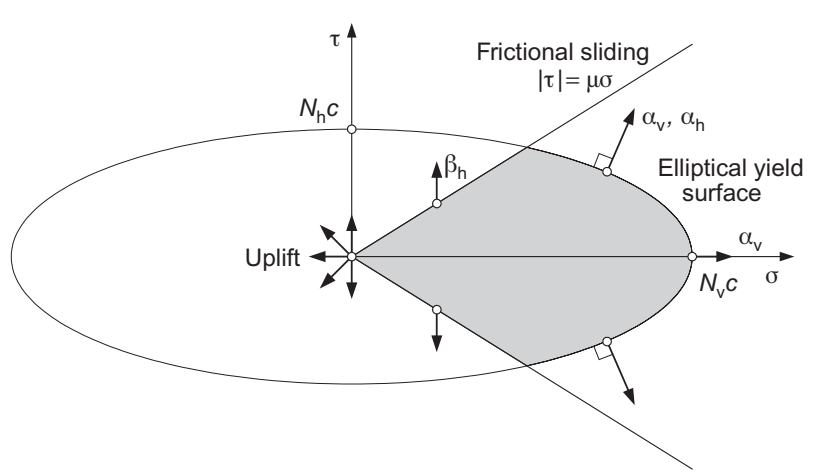

Fig. 4. Yield surfaces and flow for pointwise model venient to work with the rate-dependent formulation, for which we can define the following expressions instead of $d$ and $y$ (see Houlsby \& Puzrin, 2002, and Appendix 1). These have the effect of adding a linear viscous term to the dissipation.

$$
\begin{aligned}
& z=c \sqrt{N_{\mathrm{v}}^{2} \dot{\alpha}_{\mathrm{v}}^{2}+N_{\mathrm{h}}^{2} \dot{\alpha}_{\mathrm{h}}^{2}}+\frac{c\left(N_{\mathrm{v}}^{2} \dot{\alpha}_{\mathrm{v}}^{2}+N_{\mathrm{h}}^{2} \dot{\alpha}_{\mathrm{h}}^{2}\right)}{2 r_{1}}+\mu \sigma\left|\dot{\beta}_{\mathrm{h}}\right|+\frac{c \dot{\beta}_{\mathrm{h}}^{2}}{2 r_{2}} \\
& w=\frac{r_{1}}{2 c}\left\langle\sqrt{\left(\frac{\chi_{\alpha_{\mathrm{v}}}}{N_{\mathrm{v}}}\right)^{2}+\left(\frac{\chi_{\alpha_{\mathrm{h}}}}{N_{\mathrm{h}}}\right)^{2}}-c\right\rangle^{2}+\frac{r_{2}}{2 c}\left\langle\left|\chi_{\beta_{\mathrm{h}}}\right|-\mu \sigma\right\rangle^{2}
\end{aligned}
$$

Three-dimensional loading. To facilitate the later development of models for three-dimensional behaviour of entire footings, we state the generalisation of equations (2) and (18) to the $3 \mathrm{D}$ case. For consistency with later developments we call the stress components $\left(\sigma, \tau_{2}, \tau_{3}\right)$ and the displacement components $\left(v, u_{2}, u_{3}\right)$. The equations become

$$
\begin{aligned}
& f=\frac{k_{\mathrm{v}} G}{2 B}\left\langle v-\alpha_{\mathrm{v}}\right\rangle^{2} \\
& +\frac{k_{\mathrm{h}} G}{2 B}\left[\left(u_{2}-\alpha_{\mathrm{h} 2}-\beta_{\mathrm{h} 2}\right)^{2}+\left(u_{3}-\alpha_{\mathrm{h} 3}-\beta_{\mathrm{h} 3}\right)^{2}\right] \\
& w=\frac{r_{1}}{2 c}\left\langle\sqrt{\left(\frac{\chi_{\alpha_{\mathrm{v}}}}{N_{\mathrm{v}}}\right)^{2}+\left(\frac{\chi_{\alpha_{\mathrm{h} 2}}}{N_{\mathrm{h}}}\right)^{2}+\left(\frac{\chi_{\alpha_{\mathrm{h} 3}}}{N_{\mathrm{h}}}\right)^{2}}-c\right\rangle^{2} \\
& +\frac{r_{2}}{2 c}\left\langle\sqrt{\chi_{\beta_{\mathrm{h} 2}}^{2}+\chi_{\beta_{\mathrm{h} 3}}^{2}}-\mu \sigma\right\rangle^{2}
\end{aligned}
$$

\section{Integration over the footing}

Two-dimensional loading: strip foundation. By integrating the stress components of the single-point model over the area of the footing, a load-displacement model for the global foundation behaviour can be derived. We apply the method first to a strip footing of width $B$. A continuous distribution of single-point models integrated over the area allows calculation of the global reactions. This integration is expressed in terms of a dimensionless coordinate across the footing, $\eta=x / B$, as shown in Fig. 5, which also shows the sign conventions for force resultants and displacements of the footing.

In the model of the whole foundation, each of the following variables, employed in the pointwise model, now becomes a function of $\eta$, and we introduce the 'hat' notation

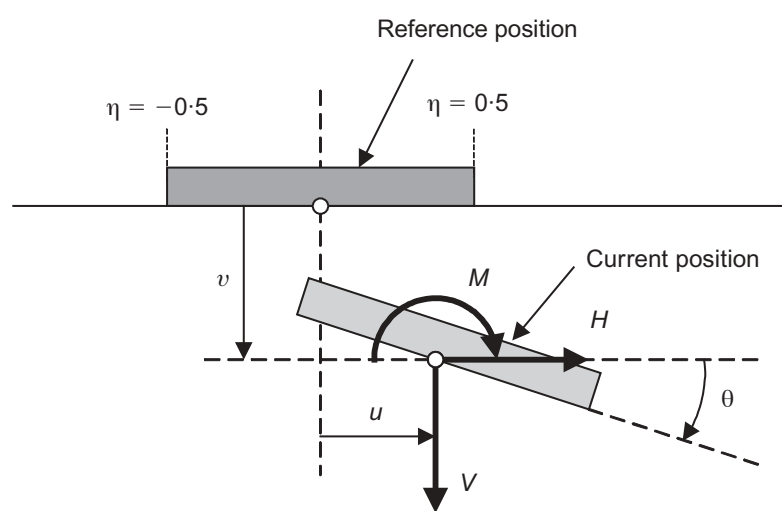

Fig. 5. Force resultants and corresponding displacements of a strip footing 
to denote any such variable: $v, u, \sigma, \tau, f, d, z, w, \alpha_{\mathrm{v}}, \alpha_{\mathrm{h}}, \beta_{\mathrm{h}}$, $\bar{\chi}_{\alpha_{\mathrm{v}}}, \bar{\chi}_{\alpha_{\mathrm{h}}}, \bar{\chi}_{\beta_{\mathrm{h}}}, \chi_{\alpha_{\mathrm{v}}}, \chi_{\alpha_{\mathrm{h}}}, \chi_{\beta_{\mathrm{h}}}, k_{\mathrm{v}}, k_{\mathrm{h}}, N_{\mathrm{v}}, N_{\mathrm{h}}$ and $\mu$. We also allow the length $L$ (perpendicular to the direction of $u$ ) of the footing to be a function of $\eta$.

The vertical and horizontal displacements at any point on a rigid footing, subjected to the global movements as shown in Fig. 5, are $\hat{v}=v+\theta x=v+B \theta \eta$ and $\hat{u}=u$.

As the total energy and dissipation now become functions of the individual functions at each point, they are now functionals, and we adopt the approach taken by Puzrin \& Houlsby (2001). Noting that an element of area of the footing is $\mathrm{d} A=\hat{L} B \mathrm{~d} \eta$, the total free energy and flow potential functionals are

$$
\begin{aligned}
& f=\int_{A} \hat{f} \mathrm{~d} A=\int_{-1 / 2}^{1 / 2} \hat{f} \hat{L} B \mathrm{~d} \eta \\
& w=\int_{A} \hat{w} \mathrm{~d} A=\int_{-1 / 2}^{1 / 2} \hat{w} \hat{L} B \mathrm{~d} \eta
\end{aligned}
$$

(In the rate-dependent formulation, which is the most useful for computation, it is convenient to work from the definition of $w$.)

The derived results are therefore

$$
\begin{aligned}
& V=\frac{\partial f}{\partial v}=\int_{-1 / 2}^{1 / 2} \frac{\partial \hat{f}}{\partial \hat{v}} \frac{\partial \hat{v}}{\partial v} \hat{L} B \mathrm{~d} \eta=\int_{-1 / 2}^{1 / 2} \frac{\partial \hat{f}}{\partial \hat{v}} \hat{L} B \mathrm{~d} \eta \\
& M=\frac{\partial f}{\partial \theta}=\int_{-1 / 2}^{1 / 2} \frac{\partial \hat{f}}{\partial \hat{v}} \frac{\partial \hat{v}}{\partial \theta} \hat{L} B \mathrm{~d} \eta=\int_{-1 / 2}^{1 / 2} \frac{\partial \hat{f}}{\partial \hat{v}} \eta \hat{L} B^{2} \mathrm{~d} \eta \\
& H=\frac{\partial f}{\partial u}=\int_{-1 / 2}^{1 / 2} \frac{\partial \hat{f}}{\partial \hat{u}} \frac{\partial \hat{u}}{\partial u} \hat{L} B \mathrm{~d} \eta=\int_{-1 / 2}^{1 / 2} \frac{\partial \hat{f}}{\partial \hat{u}} \hat{L} B \mathrm{~d} \eta
\end{aligned}
$$

Together with

$$
\hat{\chi}_{\alpha_{\mathrm{v}}}=\hat{\bar{\chi}}_{\alpha_{\mathrm{v}}}=-\frac{\partial \hat{f}}{\partial \hat{\alpha}_{\mathrm{v}}}, \hat{\chi}_{\alpha_{\mathrm{h}}}=\hat{\bar{\chi}}_{\alpha_{\mathrm{h}}}=-\frac{\partial \hat{f}}{\partial \hat{\alpha}_{\mathrm{h}}}, \hat{\chi}_{\beta_{\mathrm{h}}}=\hat{\bar{\chi}}_{\beta_{\mathrm{h}}}=-\frac{\partial \hat{f}}{\partial \hat{\beta}_{\mathrm{h}}}
$$

and (for the rate-dependent formulation)

$$
\dot{\hat{\alpha}}_{\mathrm{v}}=\frac{\partial \hat{w}}{\partial \hat{\chi}_{\alpha_{\mathrm{v}}}}, \dot{\hat{\alpha}}_{\mathrm{h}}=\frac{\partial \hat{w}}{\partial \hat{\chi}_{\alpha_{\mathrm{h}}}}, \dot{\hat{\beta}}_{\mathrm{h}}=\frac{\partial \hat{w}}{\partial \hat{\chi}_{\beta_{\mathrm{h}}}}
$$

these equations are sufficient to specify the entire footing behaviour, once specific forms of $\hat{f}$ and $\hat{w}$ are chosen. Note, however, that if $\hat{f}$ takes the particular form of equation (2), then $\bar{\chi} \alpha_{\mathrm{v}}=\sigma$ and $\bar{\chi}_{\alpha_{\mathrm{h}}}=\bar{\chi}_{\beta_{\mathrm{h}}}=\tau$.

If $\hat{L}$ is a constant, then a strip or rectangular footing can be modelled. If, on the other hand, we choose $R=B / 2$ and $\hat{L}=R \sqrt{1-4 \eta^{2}}$, we can model a circular footing of radius $R$ under planar loading.

If $\hat{k}_{\mathrm{v}}, \hat{k}_{\mathrm{h}}, \hat{N}_{v}, \hat{N}_{\mathrm{h}}$ and $\hat{\mu}$ (the parameters specifying the pointwise model) are all constants, then the resulting behaviour under vertical and horizontal loading (but in the absence of any rotation and hence moment) is identical to that of the pointwise model, simply multiplied by the area of the foundation. However, the main benefits of the approach used here are gained when these parameters are made functions of the position on the foundation. To understand the sorts of variation that are appropriate, we must consider some simple loading cases, which are explored later.

Circular footings under general loading. In the following model the footing is circular of radius $R=B / 2$. The force/ moment resultants $\left(\mathrm{V}, \mathrm{H}_{2}, \mathrm{H}_{3}, \mathrm{Q}, \mathrm{M}_{2}, \mathrm{M}_{3}\right)$ on the footing are as shown in Fig. 6, and the corresponding displacements/ rotations are $v, u_{2}, u_{3}, \omega, \theta_{2}, \theta_{3}$. Both $k$ and $N$ are allowed to

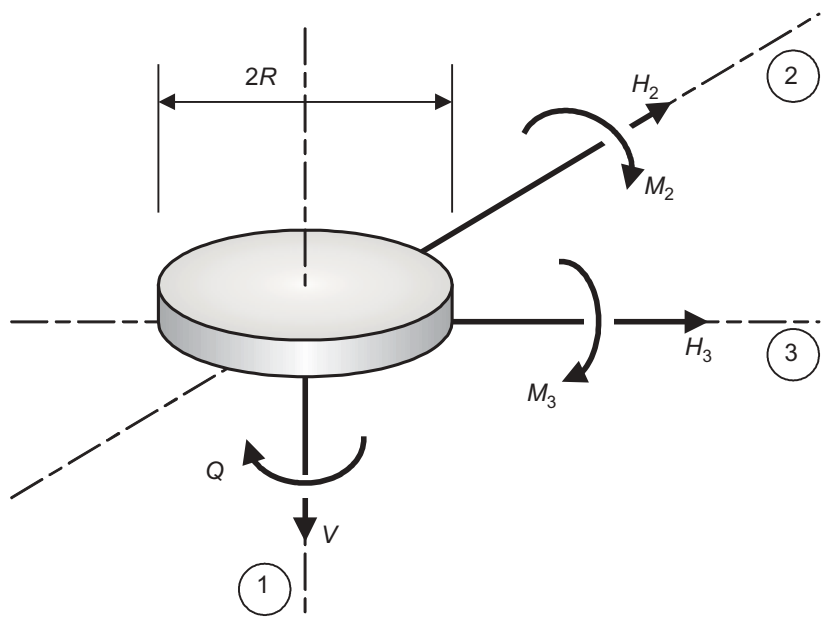

Fig. 6. Force resultants on a circular footing

vary across the foundation (as a function of radius). We adopt a non-dimensional polar coordinate system with $\eta_{r}=r / R$ and $\eta_{\phi}=\phi / 2 \pi$ (see Fig. 7). We again use a 'hat' notation to indicate any quantity that is a function of radius or of both radius and angular coordinate, e.g. $\hat{v}$. The vertical displacement at any point is

$$
\begin{aligned}
\hat{\boldsymbol{v}}\left(\eta_{r}, \eta_{\phi}\right)= & \boldsymbol{v}+x_{3} \theta_{2}-x_{2} \theta_{3}=v+\eta_{r} R \theta_{2} \cos \left(2 \pi \eta_{\phi}\right) \\
& -\eta_{r} R \theta_{3} \sin \left(2 \pi \eta_{\phi}\right)
\end{aligned}
$$

and the horizontal displacements in the 2 and 3 directions are

$$
\begin{aligned}
& \hat{u}_{2}\left(\eta_{r}, \eta_{\phi}\right)=u_{2}-x_{3} \omega=u_{2}-\eta_{r} R \omega \cos \left(2 \pi \eta_{\phi}\right) \\
& \hat{u}_{3}\left(\eta_{r}, \eta_{\phi}\right)=u_{3}+x_{2} \omega=u_{3}+\eta_{r} R \omega \sin \left(2 \pi \eta_{\phi}\right)
\end{aligned}
$$

Noting that an element of area of the circular footing is

$$
\mathrm{d} A=r \mathrm{~d} \phi \times \mathrm{d} r=R \eta_{r} 2 \pi \mathrm{d} \eta_{\phi} \times R \mathrm{~d} \eta_{r}=2 \pi R^{2} \eta_{r} \mathrm{~d} \eta_{r} \mathrm{~d} \eta_{\phi}
$$

the Helmholtz free energy and flow potential functionals are

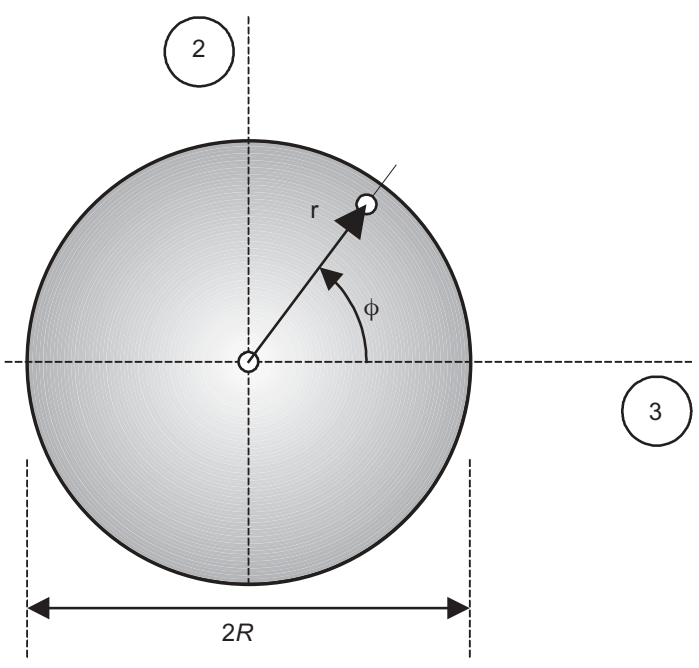

Fig. 7. Coordinate system for circular footing 


$$
\begin{aligned}
& f=\int_{A} \hat{f} \mathrm{~d} A=2 \pi R^{2} \int_{0}^{1} \int_{0}^{1} \hat{f} \eta_{r} \mathrm{~d} \eta_{r} \mathrm{~d} \eta_{\phi} \\
& w=\int_{A} \hat{w} \mathrm{~d} A=2 \pi R^{2} \int_{0}^{1} \int_{0}^{1} \hat{w} \eta_{r} \mathrm{~d} \eta_{r} \mathrm{~d} \eta_{\phi}
\end{aligned}
$$

The global response of a circular footing under general loading can be derived as

$$
\begin{aligned}
& V=\frac{\partial f}{\partial v}=2 \pi R^{2} \int_{0}^{1} \int_{0}^{1} \frac{\partial \hat{f}}{\partial \hat{v}} \frac{\partial \hat{v}}{\partial v} \eta_{r} \mathrm{~d} \eta_{r} \mathrm{~d} \eta_{\phi} \\
&=2 \pi R^{2} \int_{0}^{1} \int_{0}^{1} \frac{\partial \hat{f}}{\partial \hat{v}} \eta_{r} \mathrm{~d} \eta_{r} \mathrm{~d} \eta_{\phi} \\
& H_{2}=\frac{\partial f}{\partial u_{2}}=2 \pi R^{2} \int_{0}^{1} \int_{0}^{1} \frac{\partial \hat{f}}{\partial \hat{u}_{2}} \frac{\partial \hat{u}_{2}}{\partial u_{2}} \eta_{r} \mathrm{~d} \eta_{r} \mathrm{~d} \eta_{\phi} \\
&=2 \pi R^{2} \int_{0}^{1} \int_{0}^{1} \frac{\partial \hat{f}}{\partial \hat{u}_{2}} \eta_{r} \mathrm{~d} \eta_{r} \mathrm{~d} \eta_{\phi} \\
& H_{3}=\frac{\partial f}{\partial u_{3}}=2 \pi R^{2} \int_{0}^{1} \int_{0}^{1} \frac{\partial \hat{f}}{\partial \hat{u}_{3}} \frac{\partial \hat{u}_{3}}{\partial u_{3}} \eta_{r} \mathrm{~d} \eta_{r} \mathrm{~d} \eta_{\phi} \\
&=2 \pi R^{2} \int_{0}^{1} \int_{0}^{1} \frac{\partial \hat{f}}{\partial \hat{u}_{3}} \eta_{r} \mathrm{~d} \eta_{r} \mathrm{~d} \eta_{\phi} \\
& Q= \frac{\partial f}{\partial \omega}=2 \pi R^{2} \int_{0}^{1} \int_{0}^{1}\left(\frac{\partial \hat{f}}{\partial \hat{u}_{2}} \frac{\partial \hat{u}_{2}}{\partial \omega}+\frac{\partial \hat{f}}{\partial \hat{u}_{3}} \frac{\partial \hat{u}_{3}}{\partial \omega}\right) \eta_{r} \mathrm{~d} \eta_{r} \mathrm{~d} \eta_{\phi} \\
&==2 \pi R^{3} \int_{0}^{1} \int_{0}^{1}\left[-\cos \left(2 \pi \eta_{\phi}\right) \frac{\partial \hat{f}}{\partial \hat{u}_{2}}+\sin \left(2 \pi \eta_{\phi}\right) \frac{\partial \hat{f}}{\partial \hat{u}_{3}}\right] \eta_{r}^{2} \mathrm{~d} \eta_{r} \mathrm{~d} \eta_{\phi} \\
&=2 \pi R_{0}^{1} \int_{0}^{1} \int_{0}^{1} \frac{\partial \hat{f}}{\partial \hat{v}} \frac{\partial \hat{v}}{\partial \theta_{3}} \eta_{r} \mathrm{~d} \eta_{r} \mathrm{~d} \eta_{\phi} \\
& M_{2}= \sin \left(2 \pi \theta_{\phi}\right) \frac{\partial \hat{f}}{\partial \hat{v}} \eta_{r}^{2} \mathrm{~d} \eta_{r} \mathrm{~d} \eta_{\phi} \\
&=2 \pi R^{2} \int_{0}^{1} \int_{0}^{1} \frac{\partial \hat{f}}{\partial \hat{v}} \frac{\partial \hat{v}}{\partial \theta_{2}} \eta_{r} \mathrm{~d} \eta_{r} \mathrm{~d} \eta_{\phi} \\
&=2 \hat{f} \\
& M_{r}^{2} \mathrm{~d} \eta_{r} \mathrm{~d} \eta_{\phi}
\end{aligned}
$$

\section{MODEL CALIBRATION}

\section{Elasticity factors}

The quality of the predictions derived from the above model depends on the selection of the parameters $k_{\mathrm{v}}, k_{\mathrm{h}}, N_{\mathrm{v}}$, $N_{\mathrm{h}}$ and $\mu$. In the following we address the selection of appropriate parameters for modelling circular foundations, in which each of the above may be a function of radial position. (The model could also accommodate variation with the circumferential direction, but because of symmetry this would not normally be employed.)

Consider first the choice of $\hat{k}_{\mathrm{v}}$. If we take a constant value

$$
\hat{k}_{\mathrm{v}}=\frac{8}{\pi(1-v)}
$$

(where $v$ is Poisson's ratio), we obtain under vertical elastic loading

$$
V=\pi R^{2} \frac{\hat{k}_{\mathrm{v}} G}{2 R} v=\frac{4 G R}{1-v} v
$$

which is the standard solution for vertical loading of a rigid circular footing on an elastic halfspace (Poulos \& Davis, 1974). However, it is straightforward to show that under pure moment loading (without contact-breaking), this value results in rotational behaviour

$$
M=\frac{\pi R^{4}}{4} \frac{\hat{k}_{\mathrm{V}} G}{2 R} \theta=\frac{G R^{3}}{1-v} \theta
$$

The recognised elasticity solution for this case is, however,

$$
M=\frac{8 G R^{3}}{3(1-v)} \theta
$$

so the assumption of a constant stiffness gives a rotational stiffness too low by a factor $3 / 8$. The reason rests in the fact that the correct solutions for a rigid footing under vertical or moment loading give non-uniform distributions of stress, with singularities of stress at the edge of the footing. These singularities have a relatively higher effect on the rotational stiffness than on the vertical stiffness.

An approximate remedy is achieved by making $\hat{k}_{\mathrm{v}}$ a function of the radius. A convenient form involves a term in a power function of the radius:

$$
\hat{k}_{\mathrm{v}}=k_{\mathrm{v} 0}\left(1+f_{\mathrm{v}} \eta_{r}^{n_{\mathrm{v}}}\right)=\frac{8}{\pi(1-v)}\left[\frac{1+f_{\mathrm{v}} \eta_{r}^{n_{\mathrm{v}}}}{1+2 f_{\mathrm{v}} /\left(2+n_{\mathrm{v}}\right)}\right]
$$

where $f_{\mathrm{v}}>0$ and $n_{\mathrm{v}}>1$. The shape of this distribution is shown in Fig. 8(a). This form with a normalisation factor in the numerator is chosen so that it gives the correct vertical stiffness, but the moment stiffness is now

$$
\frac{G R^{3}}{(1-v)}\left[\frac{1+4 f_{\mathrm{v}} /\left(4+n_{\mathrm{v}}\right)}{1+2 f_{\mathrm{v}} /\left(2+n_{\mathrm{v}}\right)}\right]
$$

If both $f_{\mathrm{v}}$ and $n_{\mathrm{v}}$ are large this value approaches $2 G R^{3} /$ $(1-v)$, which is $3 / 4$ of the correct solution. Although the rotational stiffness is still too low, it is much closer to the correct solution. It is straightforward to show that (without adopting negative stiffness over part of the footing) this is as close as any model embodying the Winkler assumption can match the relative values of the vertical and moment stiffnesses. This is a fundamental weakness associated with the Winkler assumption of no interaction between points on the foundation.

A similar result is obtained for horizontal loading. If we take a constant value

$$
\hat{k}_{\mathrm{h}}=\frac{64(1-v)}{\pi(7-8 v)}
$$

we obtain under horizontal elastic loading

$$
H=\pi R^{2} \frac{\hat{k}_{\mathrm{h}} G}{2 R} u=\frac{32(1-v) G R}{7-8 v} u
$$

the standard solution for horizontal loading of a rigid circular footing on an elastic halfspace. However, under torsional loading, this value results in

$$
Q=\frac{\pi R^{4}}{2} \frac{\hat{k}_{\mathrm{h}} G}{2 R} \omega=\frac{16(1-v) G R^{3}}{(7-8 v)} \omega
$$

The recognised elasticity solution for this case is

$$
Q=\frac{16 G R^{3}}{3} \omega
$$

so the assumption of a constant stiffness gives a rotational 


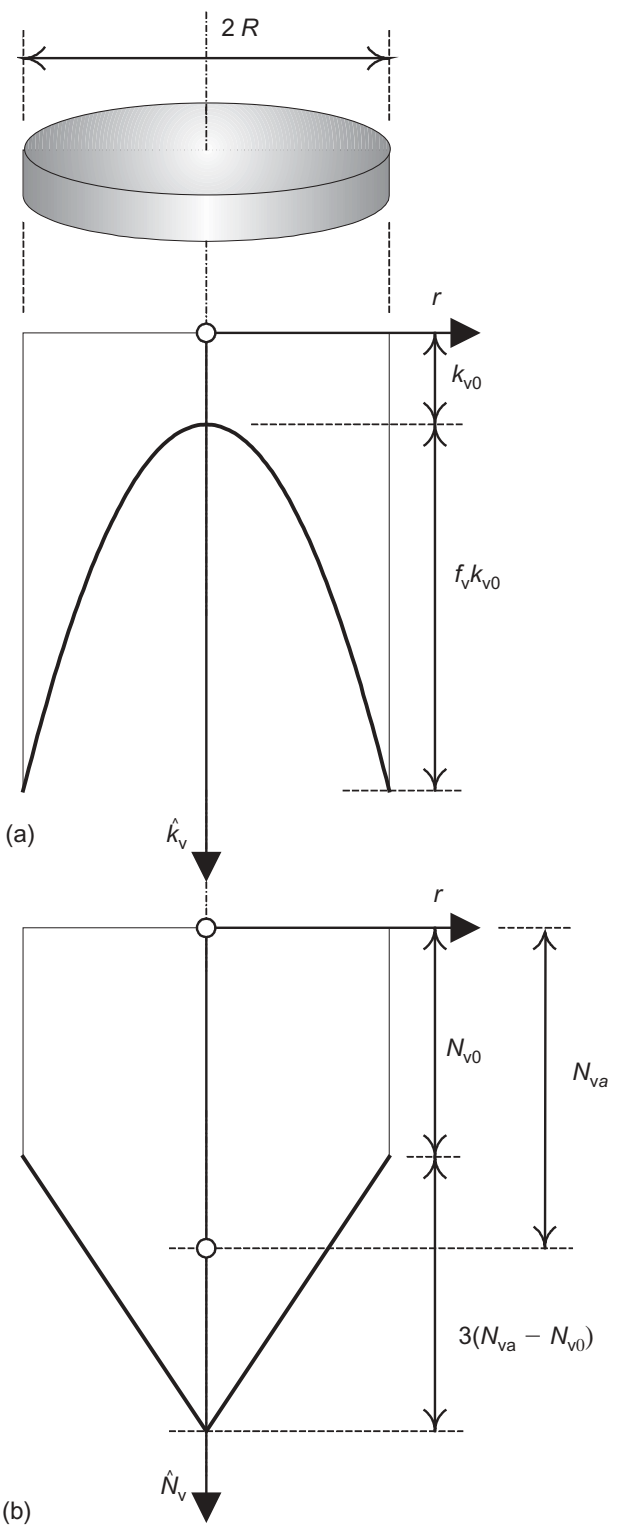

Fig. 8. Distributions of vertical stiffness and bearing capacity factors with radius: (a) elastic stiffness factor; (b) bearing capacity factor

stiffness too low by a factor $3(1-v) /(7-8 v)$, which varies from $3 / 7$ to $1 / 2$.

Again an approximate remedy is achieved by making $\hat{k}_{\mathrm{h}}$ a function of the radius. If we take

$$
\hat{k}_{\mathrm{h}}=\frac{64(1-v)}{\pi(7-8 v)}\left[\frac{1+f_{\mathrm{h}} \eta_{r}^{n_{\mathrm{h}}}}{1+2 f_{\mathrm{h}} /\left(2+n_{\mathrm{h}}\right)}\right]
$$

this gives the correct horizontal stiffness, but this time the torsional stiffness is

$$
\frac{16(1-v) G R^{3}}{(7-8 v)}\left[\frac{1+4 f_{\mathrm{h}} /\left(4+n_{\mathrm{h}}\right)}{1+2 f_{\mathrm{h}} /\left(2+n_{\mathrm{h}}\right)}\right]
$$

If both $f_{\mathrm{h}}$ and $n_{\mathrm{h}}$ are large this approaches $32(1-v) G R^{3} /(7-8 v)$, which varies from $6 / 7$ of the standard solution to the correct value at $v=0 \cdot 5$.

A limitation of the simplified model described here is that it is not capable of describing elastic cross-coupling effects between applied moment and horizontal loading (see for instance discussion by Bell, 1991).

\section{Plasticity factors}

If a constant factor $\hat{N}_{\mathrm{v}}=2+\pi \approx 5 \cdot 14$ is used, then the correct solution for vertical capacity of a strip footing on a homogeneous clay of strength $c$ is obtained. For a circular footing, a footing on non-homogeneous clay, or a footing on sand then different values are required, and in each of these cases standard plasticity solutions indicate that the vertical stress at failure increases approximately linearly from the edge of the foundation. A suitable function for $\hat{N}_{\mathrm{v}}$ for a circular footing that can capture these cases is

$$
\hat{N}_{\mathrm{v}}=N_{\mathrm{v} 0}+3\left(N_{\mathrm{va}}-N_{\mathrm{v} 0}\right)\left(1-\eta_{r}\right)
$$

as shown in Fig. 8(b), where $N_{\mathrm{v} 0} \mathrm{c}$ is the limiting vertical stress at the edge of the footing and $N_{\mathrm{va}} \mathrm{C}$ is the average stress at failure over the whole footing. For instance, for a rough circular footing on homogeneous clay of strength $c$, appropriate values would be $N_{\mathrm{v} 0}=5 \cdot 14$ and $N_{\mathrm{va}}=6.04$.

For a foundation on uniform clay of strength $c$ then a constant value $\hat{N}_{\mathrm{h}}=1.0$ would give the correct value of limiting horizontal load in the absence of a frictional cut-off. Note, however, that when the frictional cut-off is included (see Fig. 4), the maximum attainable shear stress can be calculated as

$$
\tau_{\max }=\frac{c \hat{N}_{\mathrm{h}}}{\sqrt{1+\left(\hat{N}_{\mathrm{h}} / \hat{\mu} \hat{N}_{\mathrm{v}}\right)^{2}}}
$$

For typical values $\hat{\mu} \approx 0.5$ and $\hat{N}_{\mathrm{v}} \approx 5.5$ the correct horizontal capacity is obtained by increasing the horizontal factor slightly to $\hat{N}_{h} \approx 1 \cdot 07$.

The maximum attainable values of overturning moment and axial torque are not chosen, but arise as features of the model. If the above figures are used, the maximum moment capacity is $c R^{3}\left(\frac{1}{6} N_{\mathrm{v} 0}+\frac{1}{2} N_{\mathrm{va}}\right)$ and the maximum torque is $(2 \pi / 3) R^{3} \tau_{\max }$.

Note that the dimensionless factors are calibrated for certain simple loading cases-essentially the purely elastic stiffness and the ultimate capacity for vertical and horizontal loading. The responses to overturning moment and torsion can be made to correspond reasonably well to exact solutions by allowing for distributions of the dimensionless factors across the footing base. For intermediate cases (either pre-failure non-linear response, or combinations of vertical, horizontal, moment and torsional loading) the model is not exact because of the limitations of the Winkler approach. It may, however, still capture the salient features of foundation behaviour.

\section{TEST PROBLEMS AND RESULTS}

The following examples are all computed for $R=0.5 \mathrm{~m}$, $G=200 \mathrm{kPa}, c=1.0 \mathrm{kPa}, f_{\mathrm{v}}=f_{\mathrm{h}}=5, n_{\mathrm{v}}=n_{\mathrm{h}}=4, v=$ $0.5, N_{\mathrm{v} 0}=5.14, N_{\mathrm{va}}=6.04, N_{\mathrm{h}}=1.07, \mu=0.5$ and rate dependence parameters $r_{1}=12 \mathrm{~m} / \mathrm{s}, r_{2}=0.5 \mathrm{~m} / \mathrm{s}$. The calculations used $n_{r}=n_{\phi}=16$ (see Appendix 2).

An important consequence of the increase of elastic stiffness towards the edge of the footing, coupled with the increase of vertical capacity towards the centre, is that, under pure vertical loading, yield begins at the edge of the foundation and gradually spreads inwards. This captures quite realistically what is believed to happen in practice, as the elastic stress solution for a vertically loaded rigid footing involves a stress concentration at the edge, which must cause yield in any plastic material. Fig. 9 shows the computed response for a circular loading under pure vertical loading, showing a gradual transition from elastic behaviour at small displacement to full plasticity as the bearing capacity is reached. 


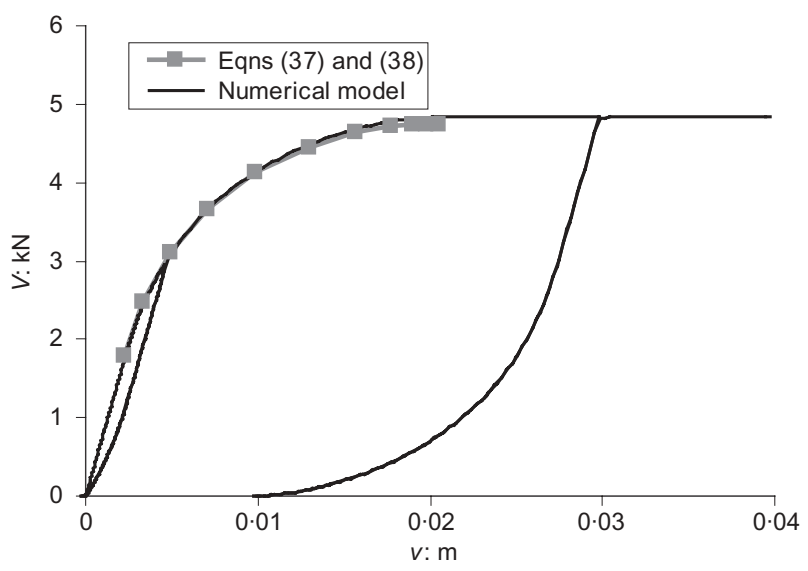

Fig. 9. Vertical load-deflection curve

The response for vertical loading can be determined analytically. After an initial purely elastic section, the elastic-plastic response can be found by assuming that within a radius $x r$ the response is elastic and outside that radius the limiting vertical stress is reached (i.e. $x$ varies from 1.0 to $0 \cdot 0$ as the load increases). The displacement can be expressed directly in terms of $x$, by making use of the displacement at which each element will yield:

$$
v=\frac{2 R c\left[N_{\mathrm{v} 0}+3\left(N_{\mathrm{va}}-N_{\mathrm{v} 0}\right)(1-x)\right]}{k_{\mathrm{v} 0} G\left(1+f_{\mathrm{v}} x^{n_{\mathrm{v}}}\right)}
$$

The load can then also be expressed in terms of $x$ by integration of the appropriate elastic and plastic expressions for the stress on the foundation:

$$
\begin{aligned}
V= & 2 \pi R^{2} \frac{k_{\mathrm{v} 0} G v}{2 R} \int_{0}^{x}\left(1+f_{\mathrm{v}} \eta_{r}^{n_{\mathrm{v}}}\right) \eta_{r} \mathrm{~d} \eta_{r} \\
& +2 \pi R^{2} c \int_{x}^{1}\left[N_{\mathrm{v} 0}+3\left(N_{\mathrm{va}}-N_{\mathrm{v} 0}\right)\left(1-\eta_{r}\right)\right] \eta_{r} \mathrm{~d} \eta_{r} \\
= & \pi R^{2}\left\{\frac{k_{\mathrm{v} 0} G v}{2 R}\left(x^{2}+2 f_{\mathrm{v}} \frac{x^{2+n_{\mathrm{v}}}}{2+n_{\mathrm{v}}}\right)+c\left[N_{\mathrm{v} 0}\left(1-x^{2}\right)\right.\right. \\
& \left.\left.+\left(N_{\mathrm{va}}-N_{v 0}\right)\left(1-3 x^{2}+2 x^{3}\right)\right]\right\}
\end{aligned}
$$

Once $x=0$ is reached then the vertical load remains

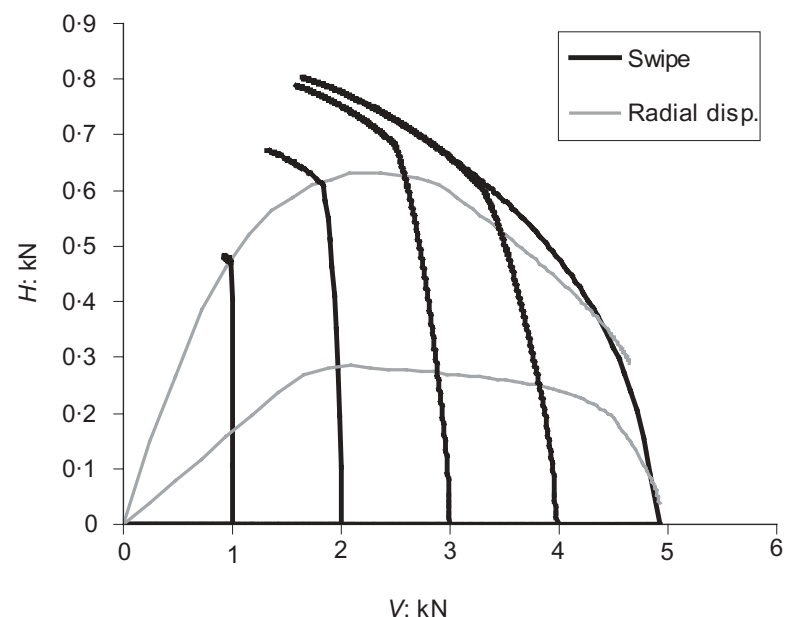

constant with depth. Fig. 9 shows the exact response computed using the above equations, demonstrating that the numerical algorithm yields an accurate solution.

On unloading after relatively small displacements the model gives an initially elastic response, followed by some non-linearity, resulting in a small hysteresis loop (Fig. 9). Unloading after very large displacements produces unrealistically large uplift of the foundation (see Fig. 9), and this is a weakness of the model.

Next we consider 'swipe' tests in which the foundation is loaded to a particular value of vertical load and then moved sideways or rotated at constant vertical displacement. These tests are commonly used in experiments to determine the shape of the yield surface for a footing (e.g. Martin, 1994; Martin \& Houlsby, 2000). Fig. 10(a) shows the load path for a horizontal swipe, and Fig. 10(b) the corresponding loaddisplacement curve. The test starting from the highest vertical load shows a very similar pattern to those shown in Fig. 11 of Martin \& Houlsby (2000), whereas those at lower vertical loads show less reduction of vertical load. Fig. 11(a, b) shows the corresponding results for moment swipes. In this case at low initial vertical load the vertical load increases during the swipe, as shown for instance in Fig. 12 of Martin \& Houlsby (2000). The ultimate moment capacity is predicted as remarkably independent of the initial vertical load.

The yield surface in load space can also be determined experimentally by tests at fixed ratios of displacements (either horizontal : vertical or rotation: vertical). The paths of such tests are also shown in Figs 10(a) and 11(a, b). In each case it is noteworthy that a constant ratio of displacement does not result in a constant ratio between the loads, but a reduction in the proportion $H / V$ or $M / V$ at larger displacements. Such tests have not been reported on clay, but Gottardi et al. (1999) report tests on sand that show a similar pattern (see their Fig. 14).

The primary purpose of this development is, however, cyclic loading. Fig. 12(a) shows the load-displacement curve for a cyclic horizontal loading test at constant vertical load, in which the amplitude of cyclic displacement is steadily increased. The stiff response at low-amplitude cycling shows a transition to a much more flexible response, coupled with considerable hysteresis, at high-amplitude cycles. Fig. 12(b) shows that the model predicts that the foundation moves downwards during each cycle of horizontal displacement. This type of behaviour could not be captured by simple plasticity models based on a single yield surface.

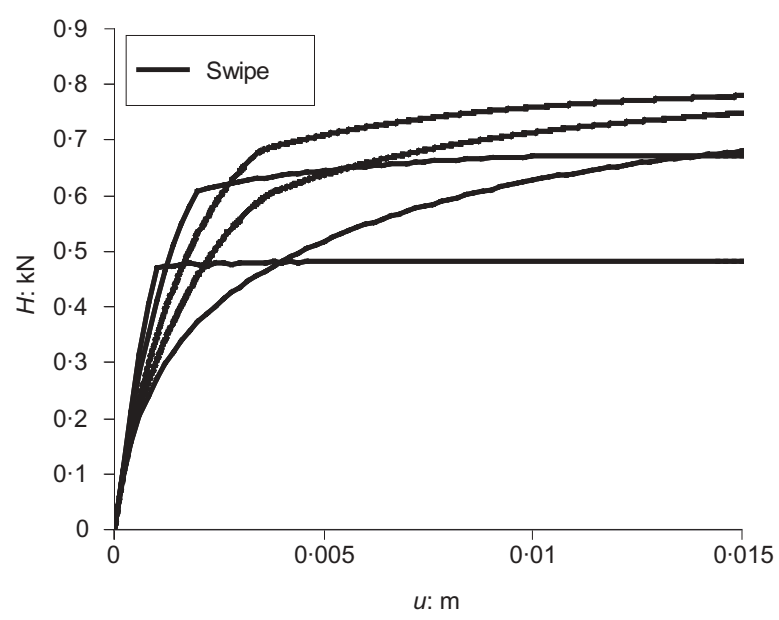

Fig. 10. Swipe and probe tests for combined $V, H$ loading: (a) load paths; (b) load-deflection response (probe tests omitted) 


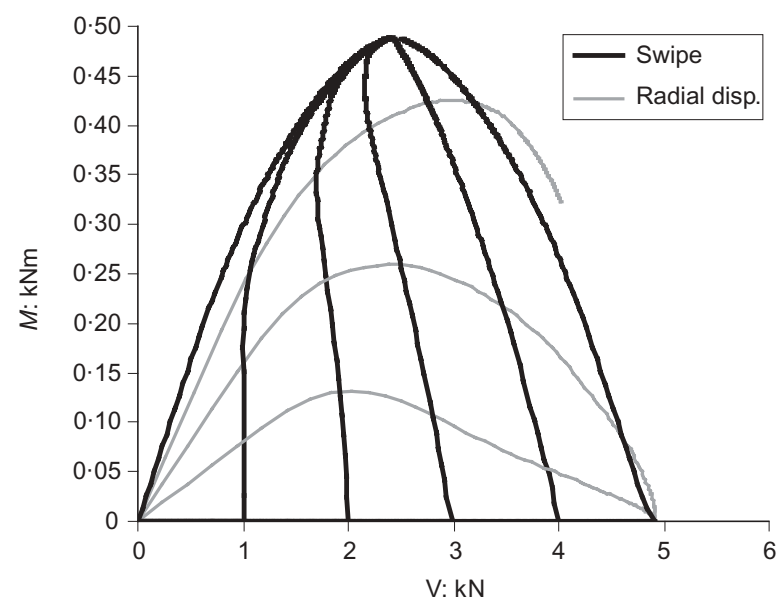

(a)

Fig. 11. Swipe and probe tests for combined $V, M$ loading:

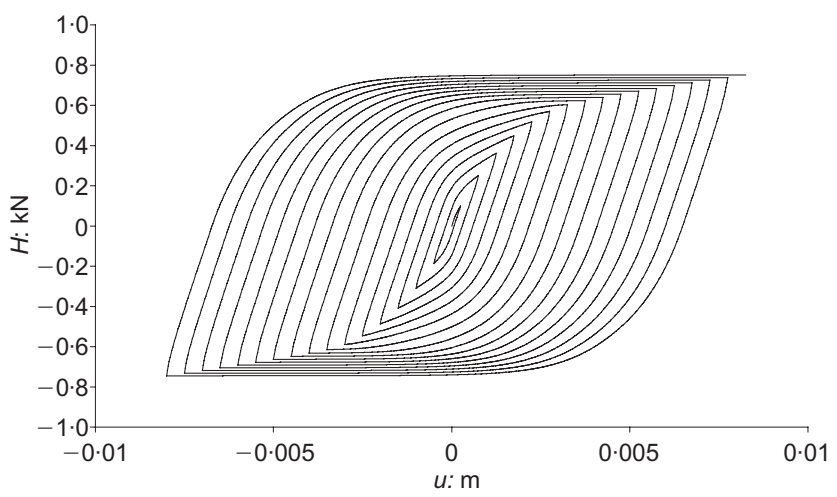

(a)

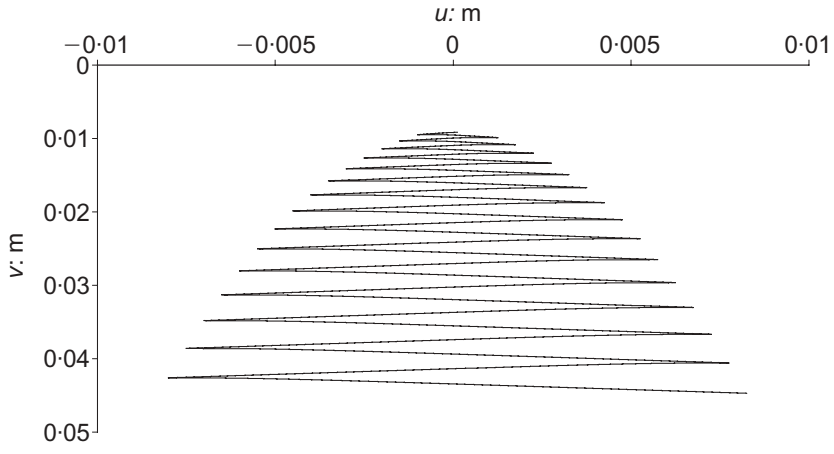

(b)

Fig. 12. Cyclic horizontal loading at constant vertical load of $5 \mathrm{kN}$ : (a) load-deflection curve; (b) vertical against horizontal displacement

Figure 13(a) shows the equivalent test with cyclic moment loading. The pattern of response is rather similar, but the shape of the hysteresis curves is now affected by the formation of gaps as contact is lost at the edges of the footing during cycling. This type of hysteresis loop has been observed, for instance, in large-scale moment tests of caissons on sand (Kelly et al., 2005), in which gapping was observed at the edge of the caisson. Fig. 13(b) shows that

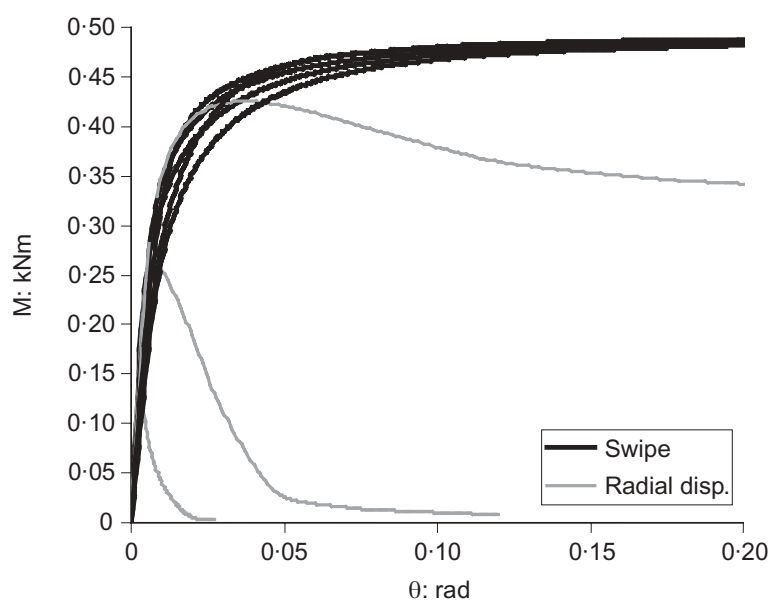

(b)

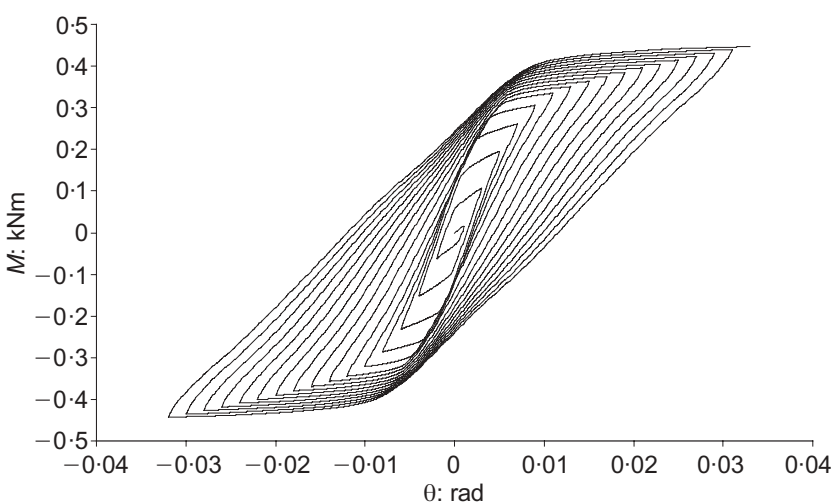

(a)

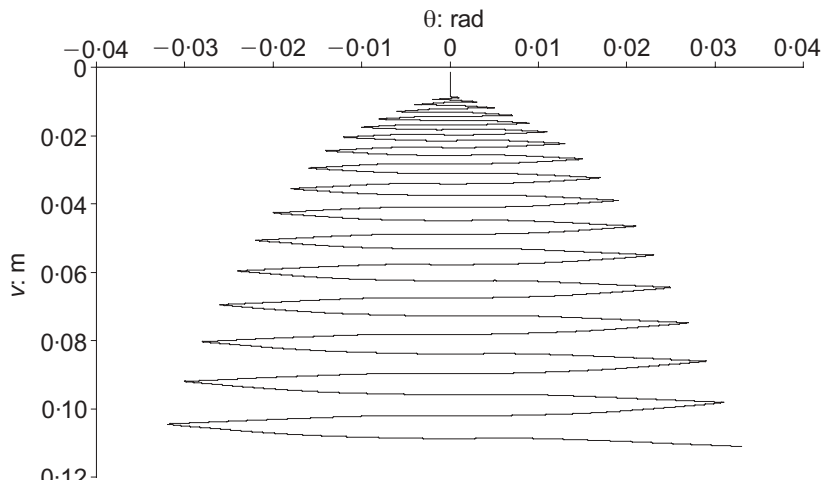

(b)

Fig. 13. Cyclic vertical loading at constant vertical load: (a) moment-rotation response; (b) vertical movement against rotation

during this cycling the model again predicts significant vertical movement of the foundation.

\section{CONCLUSIONS}

A theoretical model for cyclic loading of rigid foundations, based on the Winkler assumption, has been presented. The methodology for describing, first, the pointwise behav- 
iour of the foundation and then the integration of loads over the base of the foundation is described. The calibration procedure for the parameters describing the model is described. Finally some simple examples are presented, demonstrating the performance of the model. It is concluded that this approach provides a satisfactory basis for modelling of foundations under cyclic loading, when such models are required to be coupled to structural analyses.

\section{ACKNOWLEDGEMENTS}

This research was supported by the Department of Trade and Industry under the New and Renewable Energy Technology Programme, and the Australian Research Council through its Discovery Grant (DP0345424) and Special Research Centre programmes.

\section{APPENDIX 1. THE HYPERPLASTIC FORMULATION}

Hyperplasticity (Houlsby \& Puzrin, 2000) is an approach to plasticity theory, based on thermodynamics, in which the material response is derived from knowledge of two potential functions. The following is a brief summary of the key elements of the theory. Consider a system in which the mechanical behaviour is expressed in terms of strain $\varepsilon$ and stress $\sigma$. The 'state' of the material is also assumed to depend on 'internal variables' $\alpha$, which often (but not necessarily) play the role of plastic strain. The formulation begins with the specification of a Helmholtz free energy $f(\varepsilon, \alpha)$, and a dissipation function $d(\varepsilon, \alpha, \dot{\alpha}) \geqslant 0$, which must be a homogeneous first-order function of $\dot{\alpha}$ for a rateindependent ('plastic') material. The formulation then defines

$$
\begin{aligned}
& \sigma=\frac{\partial f}{\partial \varepsilon} \\
& \bar{\chi}=-\frac{\partial f}{\partial \alpha} \\
& \chi=\frac{\partial d}{\partial \dot{\alpha}}
\end{aligned}
$$

where $\bar{\chi}$ and $\chi$ are 'generalised stresses'. It can be shown from thermodynamics that $(\bar{\chi}-\chi) \dot{\alpha}=0$. If, however, we make the stronger assumption $\bar{\chi}=\chi$ (Ziegler's orthogonality postulate), then it follows that the entire constitutive response can be derived without any further assumptions.

The formulation allows a variety of potentials to be defined, and these are related by Legendre transforms. For instance, rather than specifying the dissipation, it is possible to specify the yield function in the form $y(\varepsilon, \alpha, \chi)=0$, and instead of equation (41) we have

$$
\dot{\alpha}=\lambda \frac{\partial y}{\partial \chi}
$$

where $\lambda$ is a multiplier that becomes determined by the consistency condition $y=0$.

In the above we are deliberately unspecific about the detailed nature of the internal variables. They can be scalars, tensors, or multiple sets of tensors. If the internal variables become an internal function (in effect an infinite number of variables), the potential functions become functionals, and the derivatives must be interpreted as Frechet derivatives (Puzrin \& Houlsby, 2001).

It is often convenient for computation to introduce some rate dependence (viscosity) into the analysis (Houlsby \& Puzrin, 2002), and in this case, rather than defining the dissipation function, we define either the force potential $z(\varepsilon, \alpha, \dot{\alpha})$, such that $\chi=\partial z / \partial \dot{\alpha}$ (instead of equation (41)), or the flow potential $w(\varepsilon, \alpha, \chi)$ such that $\dot{\alpha}=\partial w / \partial \chi$ (instead of equation (42)). These are related by $z+w=\chi \dot{\alpha}=d$. In the rate independent case, where $z$ is homogeneous and first order in $\dot{\alpha}$, then $z \equiv d$. Puzrin \& Houlsby (2003) give the extension of use of functionals to the rate-dependent case.

\section{APPENDIX 2. NOTES ON NUMERICAL IMPLEMENTATION}

In discretising the integrals in equations (29)-(34), each pointwise model is assumed to act over an equal area of the footing (i.e. $r \mathrm{~d} r \mathrm{~d} \phi$ is kept constant), and the resulting force is considered as acting through the centroid of that area. The number of increments in both $r\left(n_{r}\right)$ and $\phi\left(n_{\phi}\right)$ are specified, and the radial discretisation is calculated from

$$
\begin{aligned}
r_{i}^{2}= & \frac{i R^{2}}{n_{r}} \text { for } i=0, n_{r}, \\
& \text { and each annulus } i \text { runs from } r_{i-1} \text { to } r_{i} \\
\phi_{j}= & \frac{\pi(2 j-1)}{n_{\phi}} \text { for } j=0, n_{\phi}, \\
& \text { and each sector } j \text { runs from } \phi_{j-1} \text { to } \phi_{j} .
\end{aligned}
$$

The area of each patch is $\pi R^{2} / n_{r} n_{\phi}$, and the centroid of patch $(i, j)$ is at coordinates

$$
\frac{2 R}{3} \frac{\sin \left(\pi / n_{\phi}\right)}{\pi / n_{\phi}} \frac{\left[i^{3}-(i-1)^{3}\right]}{\sqrt{n_{r}}}, \frac{2 \pi(j-1)}{n_{\phi}} .
$$

In using the rate-dependent formulation some care is required in selection of both the time step and the ratedependence parameters $r_{1}, r_{2}$. If $r_{1}, r_{2}$ are too low then the contribution of the viscous terms becomes excessive, and if they are too large then very many time steps are required. The following procedure yields satisfactory results if a simple forward finite difference scheme is used (with out-ofbalance force correction).

(a) Select a total time increment for each phase of loading, values of $r_{1}, r_{2}$ and the number of time steps over which the loading will be applied. Carry out an initial calculation.

(b) Double the $r_{1}, r_{2}$ parameters and repeat the calculation.

(c) Repeat (b) until two successive calculations agree to within a required tolerance (a $1 \%$ difference is usually acceptable).

(d) If at any stage the calculation becomes unstable, then double the number of time steps until the calculation is stable once more.

The onset of instability in the rate-dependent calculation is very clear cut, and depends on the ratio of $r_{1}, r_{2}$ to the number of time steps, so that once the instability threshold is encountered, the steps must be increased in proportion to $r_{1}, r_{2}$.

\author{
NOTATION \\ A area \\ $B$ breadth of footing \\ $c$ soil strength parameter \\ $d$ dissipation \\ $f$ Helmholtz free energy \\ $f_{\mathrm{h}}, f_{\mathrm{v}}$ factors in stiffness variation with radius \\ $G$ soil shear modulus \\ $H$ horizontal load \\ $k_{\mathrm{h}}, k_{\mathrm{v}}$ horizontal, vertical dimensionless stiffness factors
}


$L$ length of footing

$M$ moment about horizontal axis

$N_{\mathrm{h}}, N_{\mathrm{v}}$ horizontal, vertical dimensionless capacity factors

$N_{\mathrm{v} 0}, N_{\mathrm{va}}$ value of $N_{v}$ at edge of footing and averaged over footing

$n_{\mathrm{h}}, n_{\mathrm{v}}$ exponents in stiffness variation with radius

$n_{\mathrm{r}}, n_{\phi}$ number of points in discretisation in radial and hoop directions

$Q$ torque about vertical axis

$R$ radius of circular footing

$r_{1}, r_{2}$ rate dependency parameters

$u$ horizontal displacement

$V$ vertical load

$v$ vertical displacement

$w$ force potential

$y \quad$ yield potential

$z$ flow potential

$\alpha$ internal parameter (plastic displacement)

$\beta_{\mathrm{h}}$ sliding displacement

$\eta$ dimensionless internal coordinate

$v$ Poisson's ratio of soil

$\theta$ footing rotation about horizontal axis

$\mu$ friction coefficient

$\sigma$ normal traction on footing

$\tau$ shear traction on footing

$\phi$ polar coordinate

$\chi, \bar{\chi} \quad$ generalised stress

$\omega$ twist about vertical axis

\section{Subscripts \\ $h$ horizontal \\ $r$ radial \\ $v$ vertical \\ $\phi$ hoop direction}

\section{Special functions}

$\langle x\rangle=0$ if $x<0,\langle x\rangle=x$ if $x \geqslant 0$

$\operatorname{sgn}(x)=-1$ if $x<0, \operatorname{sgn}(x)=1$ if $x>0,-1 \leqslant \operatorname{sgn}(0) \leqslant 1$

$\hat{x}$ any function of an internal coordinate $\hat{x}=\hat{x}(\eta)$

\section{REFERENCES}

Bell, R. W. (1991). The analysis of offshore foundations subjected to combined loading. MSc thesis, Oxford University.

Einav, I. (2005). Energy and variational principles for piles in dissipative soil. Géotechnique (forthcoming).

Einav, I. \& Cassidy, M. J. (2004). A variational formulation for macro modelling of rigid foundations. Int. J. Solids Struct. (submitted).

Fleming, W. G. K., Weltman, A. J., Randolph, M. F. \& Elson, W. K. (1985). Piling engineering. Glasgow: Surrey University Press.

Gottardi, G., Houlsby, G. T. \& Butterfield, R. (1999). The plastic response of circular footings on sand under general planar loading. Géotechnique 49, No. 4, 453-470.

Houlsby, G. T. \& Cassidy, M. J. (2002). A plasticity model for the behaviour of footings on sand under combined loading. Géotechnique 52, No. 2, 117-129.

Houlsby, G. T. \& Puzrin, A. M. (2000). A thermomechanical framework for constitutive models for rate-independent dissipative materials. Int. J. Plasticity 16, No. 9, 1017-1047.

Houlsby, G. T. \& Puzrin, A. M. (2002). Rate-dependent plasticity models derived from potential functions. J. Rheology 46, No. 1, $113-126$

Kelly, R. B., Houlsby, G. T. \& Byrne, B. W. (2005). A comparison of field and laboratory tests of caisson foundations in sand and clay. Géotechnique (submitted).

Martin, C. M. (1994). Physical and numerical modelling of offshore foundations under combined loads. DPhil thesis, University of Oxford.

Martin, C. M. \& Houlsby, G. T. (2000). Combined loading of spudcan foundations on clay: laboratory tests. Géotechnique 50, No. 4, 325-338.

Martin, C. M. \& Houlsby, G. T. (2001). Combined loading of spudcan foundations on clay: numerical modelling. Géotechnique 51, No. 8, 687-700.

Poulos, H. G. \& Davis, E. H. (1974). Elastic solutions in soil and rock mechanics. New York: John Wiley.

Puzrin, A. M. \& Houlsby, G. T. (2001). A thermomechanical framework for rate-independent dissipative materials with internal functions. Int. J. Plasticity 17, 1147-1165.

Puzrin, A. M. \& Houlsby, G. T. (2003). Rate dependent hyperplasticity with internal functions. Proc. ASCE, J. Engng Mech. Div. 129, No. 3, 252-263. 